\title{
The health behaviour and wellbeing of older seafarers on Merseyside - indicated changes through brief interventions
}

\author{
Malcolm John Fisk
}

De Montfort University, Leicester, United Kingdom

\begin{abstract}
Background: There is significant evidence of the poor health of seafarers that arises both from the rigours of their trade and, for many, the associated lifestyles. Such poor health can continue in later life. The objective of the research is to report on a specific project that provided brief interventions to assist older (ex-) seafarers and to establish the effect of those interventions on their knowledge, behaviours, health and wellbeing.

Materials and methods: Older seafarers were recruited to the project. Brief interventions were provided by which the knowledge of a number of older seafarers with health needs was raised about the options available to them; and the implications for their lifestyles and behaviours were addressed. Initial and final interviews were undertaken to determine any changes in self-reported health and wellbeing using both EQ5D and the Warwick-Edinburgh Mental Wellbeing Scale (WEMWBS) measures. Post project interviews took place with a sample of the older seafarers.

Results: A good level of understanding was found among the older seafarers regarding their own health. This meant that a precondition was in place, for many, by which changes in behaviours and lifestyles could take place. An important outcome was the indicated benefits of the brief interventions for self-reported wellbeing, though not statistically significant at the $95 \%$ level of confidence.

Conclusions: Endeavours within the project to reach some of those who could benefit from the brief interventions were successful. Just over half changed their behaviours or were thinking of so doing. Wellbeing gains arising were indicated.
\end{abstract}

(Int Marit Health 2017; 68, 3: 133-139)

Key words: seafarers, health, wellbeing, behaviour, brief interventions

\section{INTRODUCTION}

The Better Health for Ex-Seafarers Project (the Project) ran from 2011 to 2013. It was established in response to the outcomes of a substantial study in the United Kingdom (UK) of the health and living circumstances of seafarers and their families that had been commissioned by the Maritime Charities Funding Group [1]. The Project engaged with older seafarers within the Merseyside districts of Wirral, Liverpool and Sefton in the North West of England.

The Maritime Charities Funding Group study found that, for older seafarers, the consequences of their seafaring lives were frequently manifested in higher rates of social isolation, poverty and poor health. These consequences were noted as characterised in the self-reliant nature of seafarers (who are almost exclusively men) that can be linked, for many, to an associated disinclination to seek help when faced with needs arising from health or other challenges [2]. Associated with this may be health behaviours among some men, those in seafaring amongst them, that can be characterised by 'physical toughness, risk-taking and heavy drinking' [3]. These were noted by McVittie and McKinlay as attempts to 'enact a particular form of masculine identity' [4]. 
At the core of the Maritime Charities Funding Group study was a substantial postal survey that elicited nearly 1000 responses [1]. It was complemented by evidence from telephone interviews with staff of 18 maritime charities and focus groups with a total of nearly 100 participants in 9 English ports. The overall picture from the responses to postal survey was one in which most (82\%) older seafarers and their dependants reported a disability or illness that affected their day-to-day life. This may suggest a level of poor health that is higher than that of older people generally in the UK, the latter being noted in the study as 33\% for those aged between 65 and 75 , and almost $50 \%$ for those aged over 75 [1]. This reported level of poor health among older seafarers was considered as, at least in part, attributable to lives at sea and associated lifestyles, with social isolation noted as 'particularly prevalent' for many of the older seafarers who were single [5].

This is the context in which the Project was established. Central to the Project was the appointment of a Health Project Advisor (the Advisor). She was remitted, after some exploration of potential approaches, to recruit and make brief interventions with older seafarers who had unmet health needs in the Merseyside area; to signpost and, where appropriate, support them in accessing health services. The Advisor was accountable to a 'Core Group' comprising representatives of the Maritime Charities Funding Group, two of the three charities that funded the Project and the author of this paper (as the project evaluator). The Project also benefited from additional ideas and advice through an 'Advisory Group' of external stakeholders. The Core and Advisory Groups met, respectively on nine and four occasions in order to monitor and guide the work of the Project.

Key areas for exploration within the Project included the extent to which the activities of the Advisor would, for older seafarers

- help foster greater knowledge and understanding of their health;

- increase their preparedness to take responsibility for their health; and

- provide evidence of positive behaviour change.

Improved health and well-being, it was considered, could assist in combating the threat of social isolation (noted above as a particular risk for those who are single) and more generally to position older seafarers for greater engagement in their communities.

The Project Report written by the Advisor documented the way that her activities were balanced between a focus on individual older seafarers and on the development of links with statutory and voluntary agencies [6]. The key elements of the brief interventions were the recruitment of the older seafarers, face to face interviews, identification of sources of support (and the facilitation of links, where appropriate, to those sources); and the provision of information and advice. The Project was focused, therefore, around needs that related directly to public and preventative health. It was, in other words, primarily concerned with the effects of lifestyles and behaviours on personal health and wellbeing and the ways in which brief interventions could influence these.

\section{MATERIALS AND METHODS}

In order to be recruited to the Project the older seafarers were required to be aged 60 or more. A mix of methods was used to make contact with them including mailings by two maritime charities (Nautilus Welfare and the Shipwrecked Mariners' Society); items published in the local press; two broadcasts on Merseyside Radio; the leafleting of venues where older seafarers were known to gather (including pubs and clubs); and a specific launch event (collaborating with a parallel Caseworker project) in Liverpool.

At the end of the Project there were 35 older seafarers with whom there had been contact that could be considered as brief interventions [6]. All but 2 of the 35 were men. Almost half (49\%) lived alone. The average age was 71 (relating to an age range from 60 to 91) for whom there had been an average period of 36 years sea service. The older seafarers reported, on average, four health conditions.

With regard to the brief interventions made through the Project, these can be seen within a broader 'family' of interventions that are concerned both with informing and encouraging people regarding their health and also with nurturing (where needed) their motivation to self-manage. Interventions aimed at helping self-management have been shown to be beneficial for men [7].

Brief interventions can be linked to overlapping approaches to behaviour change that carry multiple labels. Useful guidance regarding individual-level behaviour change interventions is provided by the National Institute for Health and Care Excellence (2014). This noted that 'information is usually a necessary precursor to behaviour change' though this alone may not be enough [8]. The range of such interventions extends in a continuum from professionally delivered cognitive behavioural therapy to lay support provided through community health champions. Brief interventions fall somewhere in the middle of this continuum and can be regarded as rejecting what might be regarded as an overly medical (and, perhaps, condition focused) approaches to behaviour change. The interventions are highly sensitive to issues around health within the broader context of people's social and economic lives.

The Project was, therefore, rooted in concerns around public health - by virtue of which a wider 'real life' focus was maintained. Such interventions have been to be to some extent successful in the context of smoking cessation and reducing alcohol consumption. A precondition for that 
success has been noted as one that requires a focus on the individual rather than any specific health condition [9]. By this means the Advisor was, it was considered, better able to engage with older seafarers - and to do so outside of normal vehicles of health service 'delivery'.

\section{RECRUITMENT OF OLDER SEAFARERS}

The approach taken to recruiting older seafarers to the Project was successful in reaching at least some who had health needs but who were not initially known to the maritime charities. This is reflected in the fact that eleven of the 35 older seafarers who engaged with the Project were not already in contact with such charities. They had responded to media coverage or found out about the Project through Merchant Navy networks including the Project launch event. Six of the older seafarers were already beneficiaries of financial advice from the parallel Caseworker project and were cross-referred to the Advisor. Eight referrals were made in the other direction (to the Caseworker project).

Those who engaged with the Project had spent all or most of their seafaring lives in merchant shipping. All those recruited were registered with a general practitioner, this reflecting, in part, the biannual necessity, during their working lives, for them as merchant seamen to renew their Medical Fitness Certificate. It follows that if the Project were to have helped establish a higher degree of knowledge among older seafarers about their own health then, for many, an important pre-condition for any related behaviour change may already have been in place.

\section{FACE TO FACE CONTACT}

What became clear from the Project and, even more notably in the outcomes of the brief interventions undertaken by the Advisor, was the high value placed by older seafarers on personal contact [6]. In this context, older seafarers, albeit with good knowledge of some aspects of their health were, it appears, willing to develop new understandings and accept information, advice and guidance around their health, lifestyles and behaviour.

In order to assess the benefits of the brief interventions made, the Project included a research element by which successive face to face interviews were undertaken to establish health and wellbeing. These were complemented by ensuing personal interviews by telephone with a sample of the older seafarers concerned. Initial face to face contacts with the Advisor included, therefore, an interview to gather "base level' information. They were guided in part by a proforma and enabled the documentation, using particular measures, of self-reported health and wellbeing. The interviews normally took place in the older seafarer's home.

Two well recognised tools for self-assessment, viz. EQ5D and the Warwick-Edinburgh Mental Wellbeing Scale
(WEMWBS), were used (see below). The interviews with the Advisor were discursive and open, enabling the older seafarer to express freely their thoughts and concerns - including matters relating to their lifestyles and behaviours. The discussions (possibly a better word than interviews) served to point to some reluctance on the part of many older seafarers to change, whether then or in the past, some of the 'harmful' behaviour that was part of their social lives. But, importantly, and following from the earlier point about most older seafarers being knowledgeable about their health, it was established that many had, in prior years, changed their behaviour (notably by giving up smoking) or that they were now prepared to consider changes to their lifestyles.

The context is, therefore, one where brief interventions may, for many older seafarers, be all that is necessary to help stimulate or motivate beneficial behaviour or lifestyle changes that would help to bring about gains in health and self-reported wellbeing. This carries, however, a proviso that relates to other elements of the lives of older seafarers (e.g. around poor housing or living with debt) that may also need to be addressed.

\section{TELEPHONE INTERVIEWS WITH SERVICE USERS}

Twenty-two telephone interviews with older seafarers took place in the period from June to August 2012 and followed consent being initially obtained from them by the Advisor. They were based on a proforma that included a mix of 27 closed and open questions to gather qualitative and quantitative information relating to their views of the Project and its impact. Analysis was undertaken for answers to the closed and open-ended questions, with the latter being transcribed to help identification of themes and issues. The interviews were not recorded. Verbatim quotes that illustrated such themes and issues were included in the evaluation report [10]. The quotes reflected, in their range and content, responses to the closed questions and supplemented the information which was obtained through the use of the EQ5D and WEMWBS measures discussed below.

The interviews took place at a time that was convenient to respondents and lasted from 15 to $50 \mathrm{~min}$. The positive views of older seafarers regarding the usefulness of information conveyed was one of several themes that emerged in the course of the interviews - though it became clear that, as also noted by the Advisor herself, the Project was frequently conflated with that of the Caseworker within the parallel initiative [6].

With regard to lifestyles it was clear from the telephone interviews that the information conveyed by the Advisor was considered relevant and, in some cases, highly valuable. This was despite the good prior knowledge of older seafarers about their health conditions. All but two respondents, in fact, had accepted the advice given, with just over half (12) stating that they changed their behaviour as a consequence. 
Of the 12 older seafarers who changed behaviour as a consequence of the advice or information given by the Advisor, it can be noted that 4 consulted with health professionals and 4 talked with family and/or friends about their health or lifestyles. Where changes in behaviour had been made, these fell into just three categories: changes in diet (10), taking more exercise (6) and making reductions in alcohol consumption (3). And though not established through the telephone interviews, many respondents confirmed that they had given up smoking and/or reduced their drinking in the years prior to engaging with the Project [6]. Of the 10 who did not change their behaviour directly as a result of their initial engagement with the Advisor, 3 were thinking of changing.

What is notable is the extent to which it becomes clear that the brief intervention provided through the Project not only conveyed information on the basis of which many respondents made (or planned to make) a behaviour change, but also pointed to the extent to which the older seafarers in question were helped with regard to their motivation. Most of the older seafarers (17 or more in each case) reported that they now felt better about themselves, more optimistic and more confident. Nine of the respondents had started or re-started activities or relationships. And notable is that most (13) were using services that they had become more aware of - though these were not just those services that related to health. Most (12) professed, following their involvement with the Project, to having a more positive view of health services, with all but one feeling that they now knew where to go for 'help and advice' regarding their health.

The foregoing clearly points to the brief interventions as having stimulated behaviour change for some of the older seafarers involved or having put in place some of the preconditions for such change.

\section{MEASURES OF HEALTH AND WELLBEING}

As noted earlier, the Project used measures of (self-reported) health and wellbeing at or near the first interview. This process was repeated at a subsequent interview at or towards the end of the intervention. The measures used were EQ5D and WEMWBS. EQ5D is a European standardised measure of health developed by an international range of researchers and is validated in a now considerable range of research in the UK and beyond (see https:// euroqol.org/). In its basic form (as used in the Project) it involves self-reported measurements determined by the extent of the subject's agreement with 5 statements relating to mobility, self-care, usual activities (e.g. work, study, housework, family or leisure activities), pain/discomfort and anxiety/depression. For instance, respondents are, in respect of their self-care, invited to tick a box that affirms that they either 'have no problems with self-care', 'have some problems washing or dressing myself' or are 'unable to wash and dress myself'. In addition older seafarers are asked to rate themselves on a health rating from 0 , the 'worst', to 100, the 'best imaginable health state'.

For those older seafarers who stayed within the Project, a second exercise using these measures was conducted in the period from March to August 2012 (this being in most cases between 6 and 9 months after the first visit). To ensure the highest degree of anonymity, forms completed by the older seafarers were returned by post to the Advisor. The data gathered enabled comparisons to be made between self-reported health and wellbeing for the first set (comprising 28 or 30 of the 35 older seafarers, depending on the measure) and second set of interviews (comprising 17 or 18 of the 35 older seafarers). The data did not, however, enable changes for individual older seafarers to be measured.

Early in the Project, however, it was felt that, despite its good credentials for measuring health in terms of dependency, the EQ5D measure was narrowly focused on the practical aspects of daily living. While EQ5D might be suitable, therefore, for people with higher levels of health and support need, there were elements with which the Project was concerned relating e.g. to morale, motivation and lifestyles that were not adequately taken account of by this measure - though it did provide an overall measure of self-reported health. In order to remedy this shortcoming it was decided to supplement EQ5D with a measure on the WEMWBS. The latter was selected in recognition of it being consistently used by the North West Public Health Observatory (the region of operation of which includes Merseyside). It is, furthermore promoted by the Cheshire and Merseyside Public Health Network (CHAMPs) to help ensure consistency in outcome measures for people's mental wellbeing in the region [11].

WEMWBS was developed by an expert panel drawing on knowledge and skills ranging from psychiatry and social science to public health and health promotion [12]. It invites respondents to rate themselves according to whether 14 statements represented their feelings over the prior 2 weeks 'none of the time', 'rarely', 'some of the time', 'often' or 'all of the time'. In this instance the 14 point WEMWBS was used. Examples of the statements are 'I've been feeling close to other people' and 'I've been dealing with problems well'. All the statements on the scale are positive - this providing a consistent framework that is easily understood by respondents.

By using both EQ5D and WEMWBS the outcomes of the Project give measures that can be related to both the self-reported physical health and personal wellbeing of the older seafarers involved. They can be used as a benchmark for further work - whether with other older seafarers or in comparison with other groups of older people. 
Table 1. Overall EQ5D and WEMWBS scores

\begin{tabular}{|c|c|c|c|}
\hline & First measure & Second measure & Change \\
\hline \multicolumn{4}{|l|}{ WEMWBS - Highest score represents better health } \\
\hline I've been feeling optimistic about the future & 2.96 & 3.50 & $+18.2 \%$ \\
\hline I've been feeling useful & 3.36 & 3.47 & $+1.0 \%$ \\
\hline I've been feeling relaxed & 3.22 & 3.76 & $+16.8 \%$ \\
\hline I've been feeling interested in other people & 3.75 & 4.31 & $+4.9 \%$ \\
\hline I've had energy to spare & 2.61 & 3.00 & $+14.9 \%$ \\
\hline I've been dealing with problems well & 3.39 & 4.11 & $+21.2 \%$ \\
\hline I've been thinking clearly & 3.68 & 4.35 & $+18.2 \%$ \\
\hline I've been feeling good about myself & 3.52 & 4.06 & $+15.3 \%$ \\
\hline I've been feeling close to other people & 3.86 & 4.12 & $+6.7 \%$ \\
\hline I've been feeling confident & 3.19 & 4.06 & $+27.3 \%$ \\
\hline I've been able to make up my own mind about things & 3.96 & 4.24 & $+7.1 \%$ \\
\hline I've been feeling loved & 4.04 & 4.41 & $+9.1 \%$ \\
\hline I've been interested in new things & 3.25 & 3.94 & $+21.2 \%$ \\
\hline I've been feeling cheerful & 3.39 & 3.90 & $+16.2 \%$ \\
\hline Overall score (total) & 48.18 & 54.65 & $+13.4 \%$ \\
\hline \multicolumn{4}{|l|}{ EQ5D - Lowest score represents better health } \\
\hline Mobility & 1.77 & 1.67 & $-6.0 \%$ \\
\hline Self-care & 1.30 & 1.44 & $+10.8 \%$ \\
\hline Usual activities & 1.72 & 1.78 & $+3.5 \%$ \\
\hline Pain/discomfort & 2.07 & 1.72 & $-20.3 \%$ \\
\hline Anxiety/depression & 1.57 & 1.53 & $-2.6 \%$ \\
\hline Overall score (total) & 8.62 & 8.09 & $-6.7 \%$ \\
\hline \multicolumn{4}{|l|}{ EQ5D - Highest score represents better health } \\
\hline Overall health state & 59.63 & 64.17 & $+7.6 \%$ \\
\hline
\end{tabular}

All the figures for the older seafarers involved in the Project must be treated with caution. This reflects the fact that while most of the older seafarers interviewed for the second set of measures will have been represented among the (up to) 30 interviewed for the first set (and are, of course, both drawn from the 35 older seafarers recruited to the Project) there may be some differences in overall characteristics. It is highly likely, however, that the populations are matching (i.e. the characteristics of both groups are closely aligned) and the application of a simple statistical test (the $t$ test) to compare outcome measures is therefore, appropriate. The results of such testing are noted below in relation to the hypothesis that measures of self-reported health and wellbeing of the second sample are better than the first.

\section{RESULTS AND DISCUSSION}

In broad terms the outcomes for individual measures shown in Table 1 indicated a 14.8\% improvement in self- -reported wellbeing of older seafarers from an overall WEMWBS score of 48.18 to 54.65 during the Project.

For the WEMWBS scores the indicated pattern of reported wellbeing improvement resonates to some extent with the kind of behaviour change that the Project endeavoured to stimulate. It was, after all, concerned both to inform and to motivate older seafarers in relation to their health, their behaviours and their lifestyles. The more than $20 \%$ increase in (self) reported confidence, in dealing with problems and being interested in new things, may be relevant here. There is, at the same time, an indicated improvement of $7.7 \%$ in the self-reported EQ5D score among older seafarers for the overall health measure, this rising from 59.63 to 64.17 (Table 1). A worsening of health for 2 of the 5 measures is, however, also indicated.

In terms of statistical significance, a $t$ test undertaken in relation to the overall self-reported health and wellbeing of the two (before and after) samples finds that at the 95\% level of confidence: 
Table 2. Comparative WEMWBS scores

\begin{tabular}{llll}
\hline WEMWBS - Highest score represents better health & First measure & Second measure & North West Survey* \\
\hline I've been feeling optimistic about the future & 2.96 & 3.50 & 3.55 \\
I've been feeling useful & 3.36 & 3.47 & 3.87 \\
I've been feeling relaxed & 3.22 & 3.76 & 3.76 \\
I've been dealing with problems well & 3.39 & 4.11 & 3.99 \\
I've been thinking clearly & 3.68 & 4.35 & 4.14 \\
I've been feeling close to other people & 3.86 & 4.12 & 4.08 \\
I've been able to make up my own mind about things & 3.96 & 4.24 & 4.29 \\
Overall score & 24.45 & 27.55 & 27.70
\end{tabular}

*Relates to adults of all ages.

- for the WEMWBS scores, there is no significant change between the first and second measures; and

- for the EQ5D scores, there is no significant change between the first and second measures for either a composite measure (comprising the 5 key questions) or the overall self-reported state of health.

It is suggested, however, that a larger sample showing the same changes would have been likely to have demonstrated significance; and that this and the secondary information gathered through the follow up telephone interviews, points to positive change that may be attributed to the brief interventions. Further work with larger samples would be necessary in order to be clearer regarding this. Standing in contrast, however, is the likelihood that the brief interventions did not, overall, lead to health benefits - at least as pointed to in the EQ5D measures.

Finally, it is of some interest to make some comparisons with another study undertaken in the North West of England. This is possible by taking the 'truncated' version of the WEMWBS measure (using just 7 statements for the older seafarers) and comparing this with the results of 18,500 face to face interviews regarding mental wellbeing of adults of all ages throughout the North West of England in 2009 [13]. This finds a close concordance of scores and suggests, therefore, that the levels of self-reported health and wellbeing of older seafarers within the Project are 'in line' with the wider adult population, at least after the brief intervention. This is true for all the local authority areas in which the Project operated Wirral (27.68), Liverpool (25.69) and Sefton (27.59) (Table 2).

\section{CONCLUSIONS}

It can be concluded that the Project broadly satisfied the objectives originally set out and offered in its outcomes an indication, though without statistical significance at the $95 \%$ level of confidence, of some benefits to a range of older seafarers in their self-reported wellbeing. It was not possible, however, to point to improved health.
Relating to this it is clear that endeavours to reach some of those who could benefit from the brief interventions were successful. And regardless of the provisos above, it appears (based on the sample interviewed regarding their experience) that most older seafarers had increased knowledge or had their knowledge reaffirmed in either case with just over half changing their behaviours or thinking of so doing.

Wellbeing gains arising from the brief interventions in this Project are, it can be concluded, indicated. And it is relevant to note that the lessons learnt have been extended through training in brief intervention techniques being provided to caseworkers employed by maritime charities other than those which funded the Project. The Project has provided self-reported health and wellbeing measures that will serve as a benchmark for other studies. It raises the question, furthermore, albeit subject to the need for further investigation, as to whether such brief interventions might beneficially take place for older seafarers at an earlier point after leaving their years of sea service.

\section{ACKNOWLEDGEMENTS}

The author acknowledges the support of the Maritime Charities Group (formerly the Maritime Charities Funding Group) and of the funding bodies noted below.

\section{ETHICAL CONSIDERATIONS}

Ethical approval for the Project was deemed not required by the central National Health Service Research Ethics Committee by virtue of the work not comprising research. Key ethical principles around such matters as consent and confidentiality were fully respected during the course of the Project, its evaluation and this subsequent research.

\section{FUNDING}

The funding bodies Nautilus Welfare Fund, the Seamen's Hospital Society and TK Foundation, together with 
the Maritime Charities Funding Group agreed to the approach taken in the project and the framework for its evaluation. The author was paid for his role as independent evaluator for the Project. No funding body played a part in the gathering, inputting, analysis or interpretation of the data gathered or in the writing of this research paper.

\section{CONFLICT OF INTEREST}

The author declares that he has no competing interests.

\section{REFERENCES}

1. Maritime Charities Funding Group. Supporting seafarers and their families: Challenges for the future. Merchant Navy Welfare Board. Southampton, UK, 2007.

2. Robinson M, Robertson S, McCullagh Jo, et al. Working towards men's health: Findings from the Sefton men's health project. Health Education J. 2012; 69(2): 139-149.

3. White A, de Sousa B, de Visser R. The state of men's health in Europe. European Commission Directorate General for Health and Consumer, Brussels 2011.

4. McVittie C, McKinlay A. Help-seeking in context: Masculine and feminine identities in relation to men's health issues. Procedia Social and Behavioral Sciences. 2010; 5: 239-243, doi: 10.1016/j. sbspro.2010.07.080.
5. Fisk M, Hall D. The welfare needs of older seafarers and their (older) dependants. Insight Social Research Ltd., Abercynon and Opinion Research Services Ltd, Swansea 2007.

6. Cheater S. Better Health for Ex-Seafarers report. Nautilus International, Wallasey 2012.

7. Galdas P, Fell J, Bower P, et al. The effectiveness of self-management support interventions for men with long-term conditions: a systematic review and meta-analysis. BMJ Open. 2015; 5(3): e006620, indexed in Pubmed: 25795688.

8. National Institute for Health and Care Excellence. Behaviour change: Individual approaches. NICE Public Health Guidance 2014.

9. Harris J, Mason J, Spalding J. et al. A review of the cost-effectiveness of individual level behaviour change interventions. North West Public Health Observatory, Liverpool 2011.

10. Fisk M. Better Health for Ex-Seafarers Project: Evaluation Report. Insight Social Research Ltd, Ferndale 2012.

11. Stansfield J, Collins B, Timpson H. Using and analysing WEMWBS to measure the impact of interventions in improving mental wellbeing. Cheshire and Merseyside Public Health Collaborative, Bromborough 2013.

12. Tennant R, Hiller L, Fishwick R, et al. The Warwick-Edinburgh Mental Wellbeing Scale (WEMWBS): development and UK validation. Health Qual Life Outcomes. 2007; 5(63), doi: 10.1186/1477-7525-5-63, indexed in Pubmed: 18042300.

13. Deacon L, Carlin H, Spalding J. North West Mental Wellbeing Survey 2009. North West Public Health Observatory, Liverpool 2010. 